Boletín de la Sociedad Geológica Mexicana

VOLUMen 63, NÚM. 2, 2011, P. 171-181

\title{
Estratigrafía, análisis de secuencias y control estructural en la Formación Yegua, Cuenca de Burgos, noreste de México
}

\author{
Samuel Eguiluz de Antuñano ${ }^{1,+, *}$ \\ ${ }^{1}$ Geólogo consultor. 197 Slade Lane, Manchester, M19 2AE, Reino Unido. \\ + Dirección actual: Coordinación de Vinculación, Instituto de Geología, Ciudad Universitaria, 04510 Coyoacán, México, D. F. \\ *seguiluz662@gmail.com
}

\begin{abstract}
Resumen
La Formación Yegua (Eoceno) en la Cuenca de Burgos se divide en tres unidades. La unidad Yegua inferior es arenisca de ambiente litoral y, bajo un análisis de secuencias, representa una etapa de nivel alto, cuya cima es cortada por una superficie de discordancia o límite de secuencia regional. Esta superficie de discordancia marca un límite de secuencia de alrededor de 39.5 Ma. La unidad Yegua intermedia es una facies compleja; consiste en areniscas de abanicos de piso, canales y discordancias internas, que corresponden a un sistema de depósito bipartito compuesto por una fase de nivel bajo y una cuña progradante, además de una fase de transgresión. La unidad Yegua superior corresponde a una etapa de nivel alto y subyace a una facies de inundación que pertenece a la base de la Formación Jackson. La Formación Yegua posiblemente refleja el acarreo final de sedimento hacia el Golfo de México ancestral como resultado del levantamiento y deformación orogénica de Laramide, en el noreste de México.
\end{abstract}

Palabras clave: Estratigrafía, Cuenca de Burgos, Formación Yegua.

\begin{abstract}
The Yegua Formation (Eocene) in the Burgos Basin is divided into three units. The lower Yegua is sandstone deposited in a near shore environment of a highstand systems tract (HST), whose top is truncated by a prominent unconformity and regional sequence boundary at around $39.5 \mathrm{Ma}$. The middle Yegua is a complex facies, which consists of basin-floor fan sandstones, channels, and unconformities corresponding to a lowstand systems tract and prograding wedge, but also includes a transgressive systems tract. The upper Yegua represents a HST, which underlies the maximum flood surface that constitutes the base of the Jackson Formation. The Yegua Formation possibly reflects the final sediment influx towards the ancestral Gulf of Mexico resulting from Laramide age uplift and orogenic deformation in Northeastern Mexico.
\end{abstract}

Keywords: Stratigraphy, Burgos Basin, Yegua Formation. 


\section{Introducción}

El área de estudio se ubica en el noreste de México, en los estados de Tamaulipas y Nuevo León, con el río Bravo como límite norte. La Cuenca de Burgos pertenece a la Provincia Geológica del Golfo de México y colinda con las fajas plegadas de Sabinas y la Sierra Madre Oriental (Figura 1).

Este trabajo se basa en un estudio de los plays Yegua y Jackson realizado en Petróleos Mexicanos (archivo interno) por un equipo de trabajo bajo la dirección del autor. El trabajo que se presenta aquí tiene el propósito de describir la estratigrafía y el significado del ambiente de depósito de la Formación Yegua (Eoceno; Bartoniano Priaboniano Temprano) para contribuir al conocimiento de la evolución geológica regional. La metodología utilizada consistió en analizar las características litológicas de la Formación Yegua, tanto en superficie como en subsuelo, mediante el estudio de afloramientos, núcleos y registros de pozos, sísmica, micropaleontología de foraminíferos y la integración de trabajos publicados. En la Cuenca de Burgos no hay trabajos previos publicados sobre este intervalo estratigráfico; tampoco existen estudios de otros grupos fósiles que apoyen la interpretación de bioeventos. La edad geocronológica en millones de años (Ma) y la ubicación de las biozonas de foraminíferos son tomadas de Berggren et al. (1995) y Luterbacher et al. (2004).

\section{Estratigrafía del Eoceno}

\subsection{Litoestratigrafía}

La litoestratigrafía del Eoceno de la Planicie Costera de Texas y Cuenca de Burgos está basada en el estudio de afloramientos descritos desde finales del siglo XIX y principios del siglo XX. La división estratigráfica incluye, de la base a la cima, los grupos Wilcox y Claiborne y la Formación Jackson (Plummer, 1932). El Grupo Claiborne se subdivide en los subgrupos Mount Selman y Cook Mountain. El primero está constituido, de la base a la cima, por las formaciones Reklaw, Queen City y Weches y el segundo, por la Formación Sparta en la base y la Formación Crockett en la cima (Figura 2). La Formación Carrizo es la unidad estratigráfica más antigua del Grupo Claiborne, mientras que la Formación Yegua es la unidad más joven. Esta última formación sobreyace a la unidad Crockett y subyace a los estratos Jackson o Moodys Branch. La Formación Yegua es equivalente a la Formación Cockfield en Luisiana.

El Grupo Claiborne que aflora en Texas fue dividido por Kennedy (1892) en dos partes. La unidad inferior incluyó desde la Formación Carrizo hasta la Formación Weches, y puede correlacionarse sin dificultad desde el oriente hasta el suroccidente de Texas. La parte superior del Grupo Claiborne (serie Cook Mountain) nuevamente incluyó a los

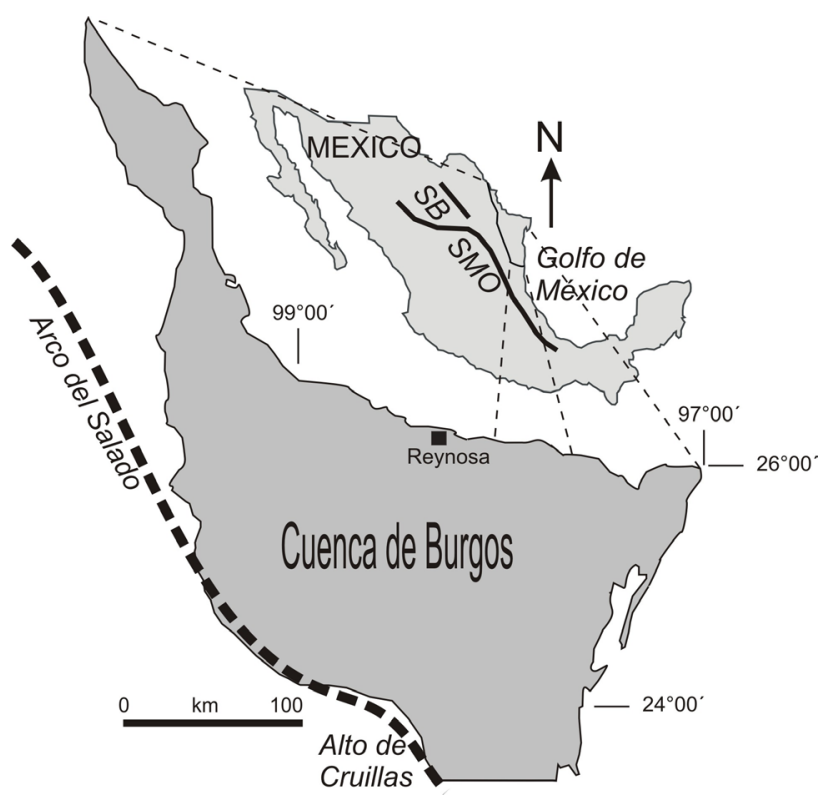

Figura 1. Localización del área de estudio en la Cuenca de Burgos y su relación con las fajas plegadas de la Sierra Madre Oriental (SMO), Sabinas (SB) y Arco del Salado-Cruillas.

estratos Weches en la base y, en la parte media y la cima, los estratos Sparta y Crockett, presentes en el centro y oriente de Texas, pero en el suroccidente de Texas es difícil darles seguimiento en superficie. La dificultad consiste en que el miembro arenoso basal (Sparta) de esta división superior está ausente. La división de Kennedy (1892) carece de claridad y Dumble (1894) se apoyó en esa estratigrafía para modificar su definición original para la Formación Yegua. La situación referida ocasionó que en el suroccidente de Texas quedara indiferenciado el miembro superior del Grupo Claiborne (Plummer, 1932).

Penrose (1890) nombró como Fayette a la sección de estratos que sobreyacen a la Formación Crockett. Kennedy (1892) designó como depósitos Lufkin a las capas más jóvenes del Grupo Claiborne y en el mismo año Dumble (1892) consideró como Yegua a los mismos estratos referidos como Fayette por Penrose (1890). Posteriormente, Dumble (1894), apoyado en los trabajos de Kennedy (1892), desafortunadamente incluyó dentro de los estratos no marinos de Yegua a algunos estratos marinos de la Formación Crockett y creó una confusión que ha permanecido vigente hasta nuestros días. En esta imprecisión también estuvieron las descripciones realizadas por Kennedy (1895), al incluir dentro de la Formación Yegua estratos de las formaciones Crockett y Sparta. Simultáneamente, Vaugham (1896) nombró como "Cocksfield Ferry" en Luisiana a estratos aproximadamente equivalentes a la Formación Yegua original de Dumble (1892). Posteriormente, Deussen (1914) restringió el uso de Formación Yegua (como inicialmente lo propuso Dumble, 1892) para nombrar solamente a los estratos no marinos ubicados entre las capas marinas Claiborne, abajo, 


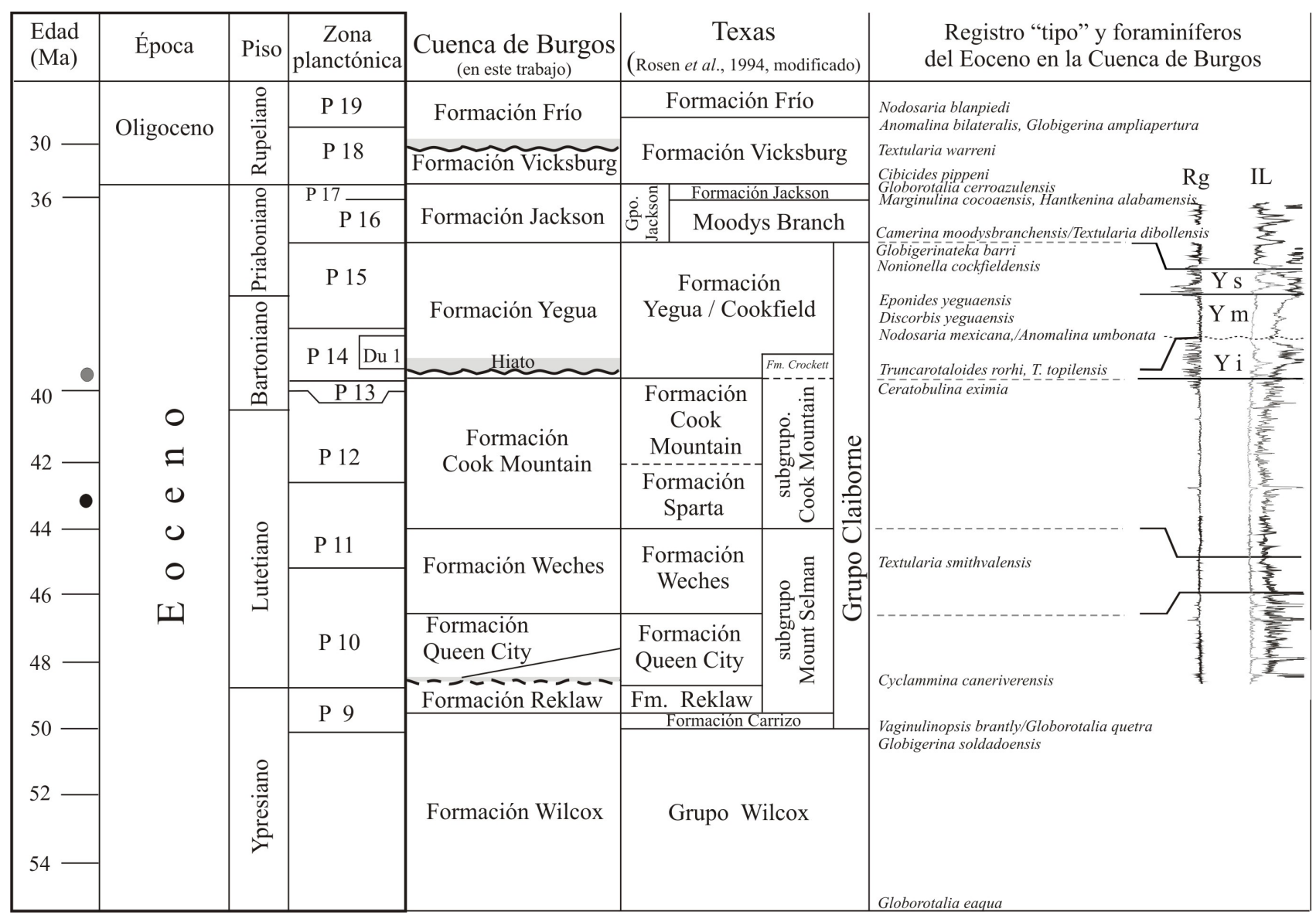

Figura 2. Nomenclatura litoestratigráfica del Eoceno entre la Cuenca de Burgos y Texas. La escala geocronológica en millones de años (Ma) propuesta para los pisos y épocas (Berggren et al., 1995), se vincula con la las biozonas planctónicas y de mamíferos tomada de Luterbacher et al. (2004) y se ajustó al contenido fosilífero de las formaciones para la Cuenca de Burgos (Segura Treviño et al., 2006) y Texas (Rosen et al., 1994; Holroyd, 2002). El registro tipo (curvas de rayos gamma a la izquierda y de resistividad a la derecha), muestra las características geofísicas de la Formación Yegua y sus unidades inferior, media y superior y su contacto con las formaciones Jackson y Cook Mountain. Los puntos gris y blanco en la escala de millones de años corresponden con la edad isotópica para rocas deformadas y no deformadas, respectivamente, del evento de edad Laramide en la Cuenca de Sabinas (Chávez-Cabello, 2005); el punto negro representa levantamiento temprano de la Sierra Madre Oriental (Gray et al., 2001).

y las capas Jackson, arriba, e indicó la equivalencia de la Formación Yegua con las capas Cocksfield Ferry definidas por Vaugham (1896). Divisiones menores de la parte superior del Grupo Claiborne fueron rechazadas por el Servicio Geológico de los Estados Unidos (Plummer, 1932).

Para resolver las dificultades estratigráficas en el Grupo Claiborne del suroccidente de Texas, Eargle (1968) propuso usar los nombres Laredo y Pico (este último propuesto previamente por Gardner, 1938, en Eargle, 1968) como equivalentes de la Formación Sparta, pero esta proposición no fue aceptada. En el suroeste de Texas, los trabajos geológicos resolvieron usar el nombre de Cook Mountain para los estratos que sobreyacen a la Formación Weches y designar como Yegua a las capas comprendidas entre las formaciones Jackson y Cook Mountain; por consiguiente, el nombre Crockett está en desuso y la Formación Yegua incluye a estratos tanto marinos como continentales.

La estratigrafía del suroccidente de Texas fue extrapolada a la Cuenca de Burgos, en donde es aplicada, con algunas variaciones, por geólogos de la industria petrolera de México. La división estratigráfica del Eoceno en la Cuenca de Burgos está compuesta por las unidades Reklaw, Queen City, Weches, Cook Mountain, Yegua y Jackson. A la unidad Carrizo se le incorpora en los estratos Wilcox, mientras que el Grupo Claiborne y el subgrupo Mount Selman han caído en desuso. El nombre Cook Mountain se emplea como formación, mientras que los nombres Pico, Sparta, Laredo y Crockett no se reconocen. La Formación Yegua, hasta antes de este trabajo, se había mantenido como una unidad indivisible, mientras que Grupo Jackson se usa en la Cuenca de Burgos con el rango de formación y se divide informalmente en tres miembros, inferior, medio y superior (Figura 2).

Desafortunadamente, la imprecisa definición de los grupos y formaciones en Texas fue aplicada en México, lo que dificulta hacer correlaciones entre ambas áreas y comprender la evolución geológica del noreste de México de manera integral. 
El nombre de Formación Yegua en la Cuenca de Burgos tiene dificultades en su uso por varias razones. La primera consideración es el resultado de adoptar una nomenclatura imprecisa que sigue la redefinición de Dumble (1894), quien incluyó estratos arenosos marinos de la Formación Crockett, con características litológicas diferentes, dentro de los estratos continentales de la Formación Yegua. Otra inconsistencia resulta de la muy restringida presencia de capas continentales de esta formación, tanto en la superficie como en el subsuelo, en la Cuenca de Burgos. Las capas comprendidas entre las unidades estratigráficas Cook Mountain y Jackson son depósitos litorales o marinos francos, con diferentes litologías. Las capas continentales están muy restringidas en superficie y las capas continentales del miembro medio de la Formación Jackson, que afloran en las cercanías del vertedero de la Presa Marte R. Gómez, Tamaulipas, fueron referidas inapropiadamente como la Formación Yegua. Una tercera consideración es que al incluir los estratos de la Formación Crockett dentro de la Formación Yegua, el subgrupo Cook Mountain no puede ser subdividido y, por lo tanto, es relegado a un estatus de formación, mientras que la Formación Yegua permanece con su mismo estatus. Antes de este estudio, en la Formación Yegua no se distinguían sus diferentes ambientes de depósito. Por lo anterior, el nombre Yegua ha sido usado para incluir capas ubicadas por su posición estratigráfica y no por sus características litológicas.

\subsection{Bioestratigrafía}

La bioestratigrafía del intervalo Eoceno está dada por foraminíferos bentónicos y planctónicos, que varían según su posición relacionada con la distribución de facies dentro de las secuencias de depósito de la planicie costera del oeste del Golfo de México. En la Cuenca de Burgos no existen estudios de otros grupos fósiles que colaboren con la interpretación de bioeventos.

De acuerdo a varios autores (Edwards, 1990; Rosen et al., 1994; Ewing y Vincent, 1997b; Ewing, 2007), la base del Grupo Jackson, representado por la Formación Moodys Branch, contiene la presencia de Globigerinatheka semiinvoluta, que está asociada, de acuerdo a su ambiente de depósito, con Textularia dibollensis, Camerina moodybranchensis, Glandulina laevigata, Anomalina hemisphaerica y A. perforata. Para Rosen et al. (1994), el conjunto bioestratigráfico puede situarse en parte de la zona planctónica P 16. Luterbacher et al. (2004) ubican la base de la biozona P 16 en $35.2 \mathrm{Ma}$, que pertenece al piso Priaboniano (Eoceno Tardío). En la Cuenca de Burgos, el conjunto de Globigerinatheka semiinvoluta (Keijzer), con Camerina moodybranchensis y Textularia dibollensis Cushman, se presentan en el miembro inferior del Grupo Jackson (Figura 2) y se ubican en la biozona P 16 (Segura et al., 2006).

La Formación Yegua en este trabajo se divide en tres unidades litoestratigráficas. Las unidades superior y media contienen Nonionella cockfieldensis, Globigerinatheka barri, seguidas de Discorbis yeguaensis, Eponides yeguaensis Cushman y Nodosaria mexicana; en la base son más comunes Anomalina umbonata y Globigerina yeguaensis Applin. Este conjunto de foraminíferos representa la biozona P 15 y parte de la biozona P 14. La base de la unidad Yegua intermedia descansa sobre una prominente discordancia regional y contiene foraminíferos retrabajados que proceden de la base de la biozona P 14 . La mezcla de conjuntos faunísticos puede confundir e interpretarse de maneras inconsistentes. La unidad inferior de Yegua, que subyace a la discordancia regional, se caracteriza por su contenido de Truncorotaloides rohri, $T$. topilensis, los que a su vez sobreyacen a Ceratobulimia eximia (biozona P 14), foraminífero característico de la Formación Cook Mountain (Figura 2).

Rosen et al. (1994) indican que en Texas y Luisiana la Formación Yegua puede dividirse en una unidad superior y otra inferior. La unidad superior está representada por abundancia de Globigerinatheka barri y, en la zona de inundación, la presencia de Nonioella cockfieldensis y escaso Discorbis yeguaensis, seguida por Eponides yeguaensis y E. mexicana, que ocurren en la cuña progradante de nivel bajo de esta secuencia, mientras que en el borde de la plataforma se presentan de forma común Gyroidina octacamerata y Bathysiphon eocénica. Rosen et al. (1994) señalan que Nodosaria mexicana aparece normalmente abajo de Eponides yeguaensis cuando la cuña progradante se expande. Según esos autores (Rosen et al., 1994) el conjunto de foraminíferos representa la biozona P 15 y agregan que en esta unidad superior la abundancia de formas bentónicas arenosas da idea de una rápida tasa de depósito. Para Swenson (1977) y Rosen et al. (1994) la unidad inferior de la Formación Yegua está representada por la presencia de Anomalina spp. y Eponides yeguaensis; menos comunes son Eponides guayabalensis y Nodosaria mexicana, con abundancia de Anomalina umbonata en la sección condensada de la superficie de máxima inundación de esta secuencia. Rosen et al. (1994) calibran a esta unidad inferior con Truncorotaloides rhori, Globorotalia lehneri y Globorotalia renzi. Además mencionan que Ceratobulimia eximia puede ocurrir raramente en la parte inferior de esta sección y concluyen que todas las faunas de la unidad inferior de Yegua en Texas y Luisiana corresponden a la parte superior de la biozona P 14, mientras que la parte inferior de esta biozona contiene Clavulinoides guayabalensis y Truncorotaloides topilensis asociados a Globorotalia spinulosa y G. bullbrooki, este último contenido en una segunda unidad condensada y representativo de la biozona P 13.

En el presente trabajo se considera que el conjunto Anomalina/Truncorotaloides rhori-T. topilensis puede corresponder en tiempo con el depósito marino de la Formación Crockett, cuyas capas superiores fueron tomadas por Dumble (1894) como la base de su redefinida Formación Yegua, mientras que para Rosen et al. (1994) corresponden 
a la base de lo que refieren como Yegua inferior.

En la Cuenca de Burgos, la cima de la Formación Cook Mountain es arcillosa y representa una superficie de inundación. Se caracteriza por la presencia de Ceratobulimia eximia, mientras que Orbitolinoides beckmanni (biozona $\mathrm{P}$ 13) y Asterigerina texana (biozona P 12) se encuentran en la parte inferior de esta formación (Figura 2). Los estratos de Cook Mountain sobreyacen a la Formación Weches, que se asigna a la biozona $\mathrm{P} 11$ por la presencia de Textularia smithvallensis (Rodríguez-Lozano, 1999).

Los conjuntos de foraminíferos descritos para la Formación Yegua en la Cuenca de Burgos y en Texas se sitúan en el Eoceno medio y tienen como característica la presencia de biozonas subtropicales y tropicales (LivasVera, comunicación personal). Luterbacher et al. (2004) colocan la base de la biozona P 15 en $38.0 \mathrm{Ma}$, la de P 14 en $39.6 \mathrm{Ma}$, y la de P 13 en $40.8 \mathrm{Ma}$, todas ellas en el Bartoniano, mientras que la base de la biozona P 12, en 43.6 $\mathrm{Ma}$, pertenece al Lutetiano.

En este estudio, la discordancia regional en la base de la unidad Yegua medio está determinada por el conjunto paleontológico y la posición estratigráfica. Esta discordancia es un prominente límite de secuencia correlativo con el cambio del nivel del mar propuesto por Haq et al. (1988) en 39.5 Ma. La resolución entre los datos de paleontología e isotopía impide una mayor definición para ubicar la edad de esta discordancia.

Lagoe y Layman (1994) identifican en la Formación Yegua de Texas y Luisiana cinco biofacies mayores representadas, de la base a la cima, por nerítico externo
(Uvigerina spp.), nerítico medio (Eponides mexicanus), nerítico interno a medio (Textularia spp.), nerítico interno (Forilus bantkeni), y marino marginal (Ammobaculitas hockleyensis). En la Cuenca de Burgos, la asociación de Anomalina/Truncorotaloides rhori representa ambientes nerítico interno a nerítico medio. El sistema de depósito está truncado por una discordancia; sobre ésta hay ambientes que se profundizan variando de marino marginal a nerítico medio, representados por Nodosaria/Discorbis y Gyroidina sp./Eponides sp., repitiendo un ambiente somero en la cima con la presencia de Nonionella sp.

Holroyd (2002) reporta la presencia de un vertebrado antracotérido (Artiodactyla: Mammalia), género Heptacodon, en la Formación Yegua en Texas. Esta especie parece ser la más primitiva de las cuatro especies conocidas de Heptacodon. Este organismo terrestre es ubicado en el Eoceno Medio tardío (periodo Duchesneano de las edades de mamíferos terrerstres de Norteamérica; Luterbacher et al., 2004). La posición de este fósil en la biozona Du 1 se correlaciona con la biozona P 14 (38.2 a 39.3 Ma, Figura 2). Heptacodon, en adición a los organismos planctónicos, confirma una regresión y el paso estratigráfico entre estratos marinos y continentales de la Formación Yegua.

\section{Presentación de los datos}

La correlación de las secciones regionales transversales a la cuenca (Figuras 3, 4 y 5) muestra un marco geológico ilustrativo en ellas. Al poniente, las secciones muestran

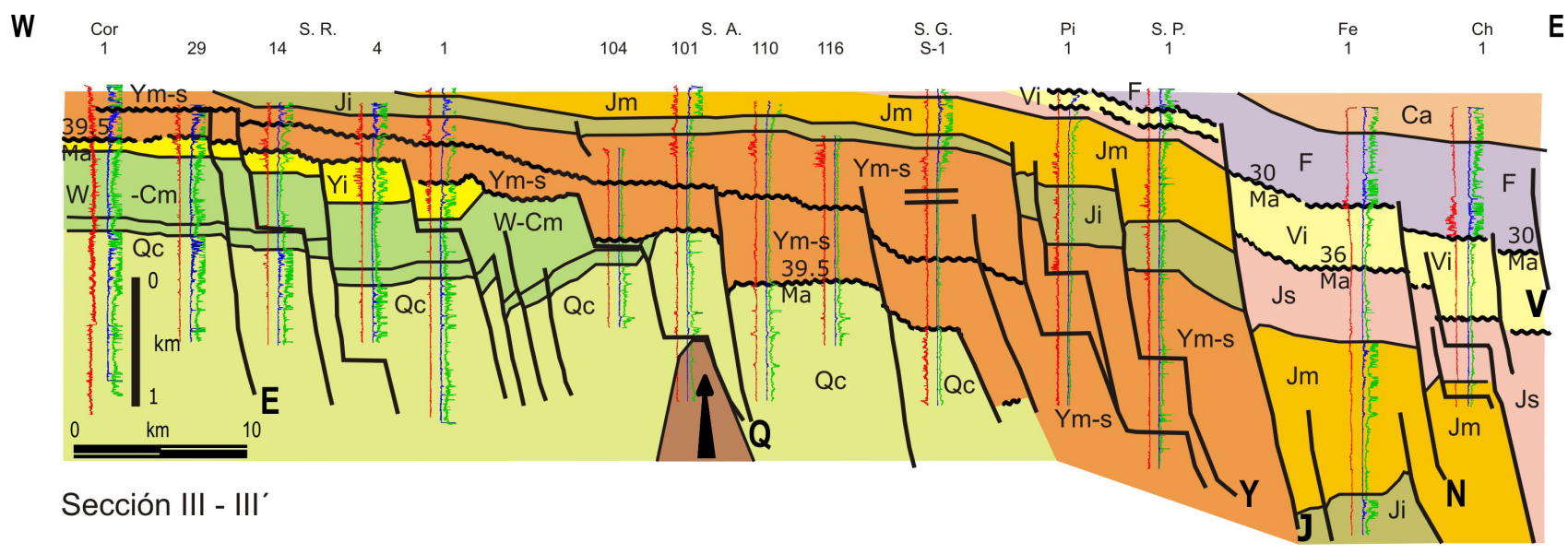

Figura 3. Leyenda para las secciones regionales orientadas de oriente (E) a occidente (W). Formaciones: Queen City (Qc); Weches y Cook Mountain (agrupadas W-Cm), Yegua inferior (Yi), Yegua intermedia y superior (Ym-s), Jackson inferior (Ji), Jackson medio (Jm), Jackson superior (Js), Vicksburg (Vi), Frío indiferenciado (F), Catahoula (Ca). Fallas de expansión: Eoceno (E), Yegua (Y), Jackson (J), Vicksburg (V); otras fallas importantes son M y N. La flecha gruesa corresponde con inyecciones de arcilla. Curvas de registros: Potencial espontáneo y/o rayos gama a la izquierda de los pozos en color rojo; resistividad a la derecha de los pozos en colores azul (0-20 $\Omega / \mathrm{m})$ y verde (0-4 $\Omega / \mathrm{m})$. Límites de secuencia mayores (SB): $39.5 \mathrm{Ma}$, $36 \mathrm{Ma}$ y 30 $\mathrm{Ma}$; dentro de Yegua medio hay varias discordancias internas. Nótese entre las secciones que la Formación Jackson superior al poniente descansa sobre Yegua intermedio-superior, pero al norte y oriente, está sobre los bloques estructuralmente hundidos; Jackson Superior descansa concordantemente sobre Jackson Medio. En el sur del área la Formación Vicksburg descansa discordantemente (36 Ma) sobre Jackson Superior, o incluso erosiona a Jackson Medio, y Frío descansa invariablemente de forma discordante (30 Ma) sobre Vicksburg. En la Figuras 7 está la ubicación de las secciones mostradas en este trabajo y otras secciones de apoyo adicionales. Los símbolos de letras y números en la parte superior de las secciones identifican a los pozos citados en el texto. Identificación de pozos modificada por obvias razones. 
W

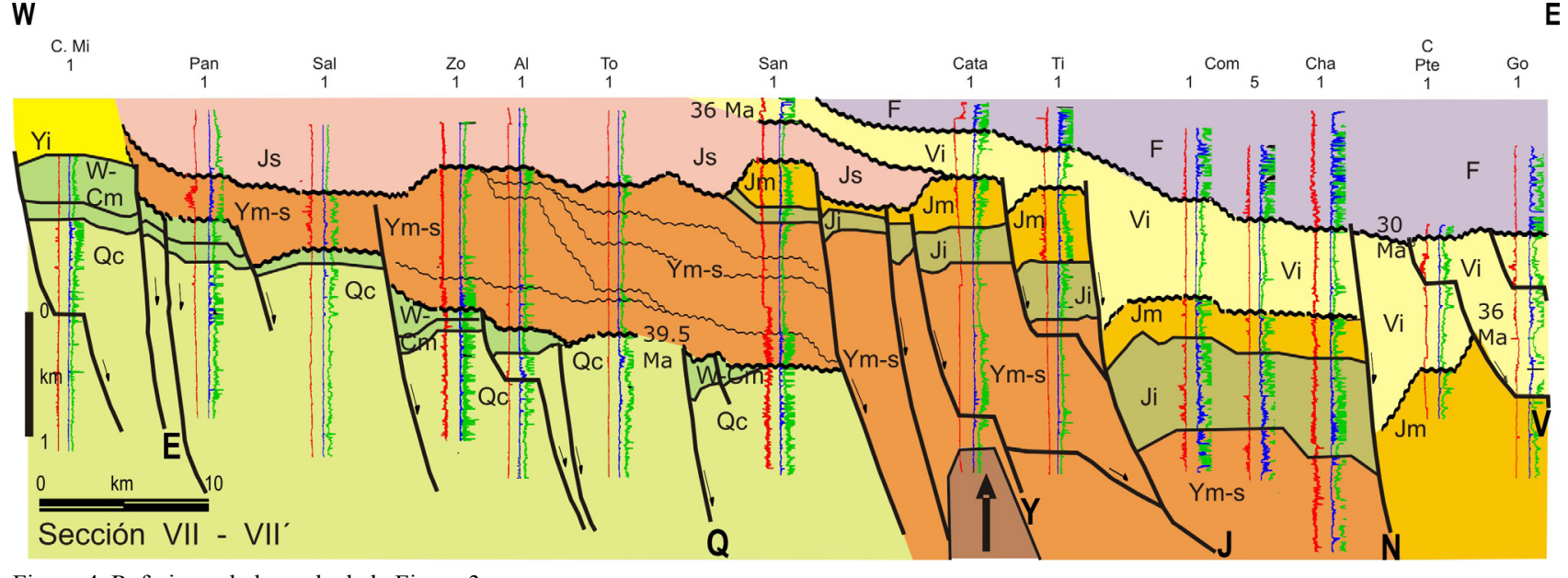

Figura 4. Referirse a la leyenda de la Figura 3.

W

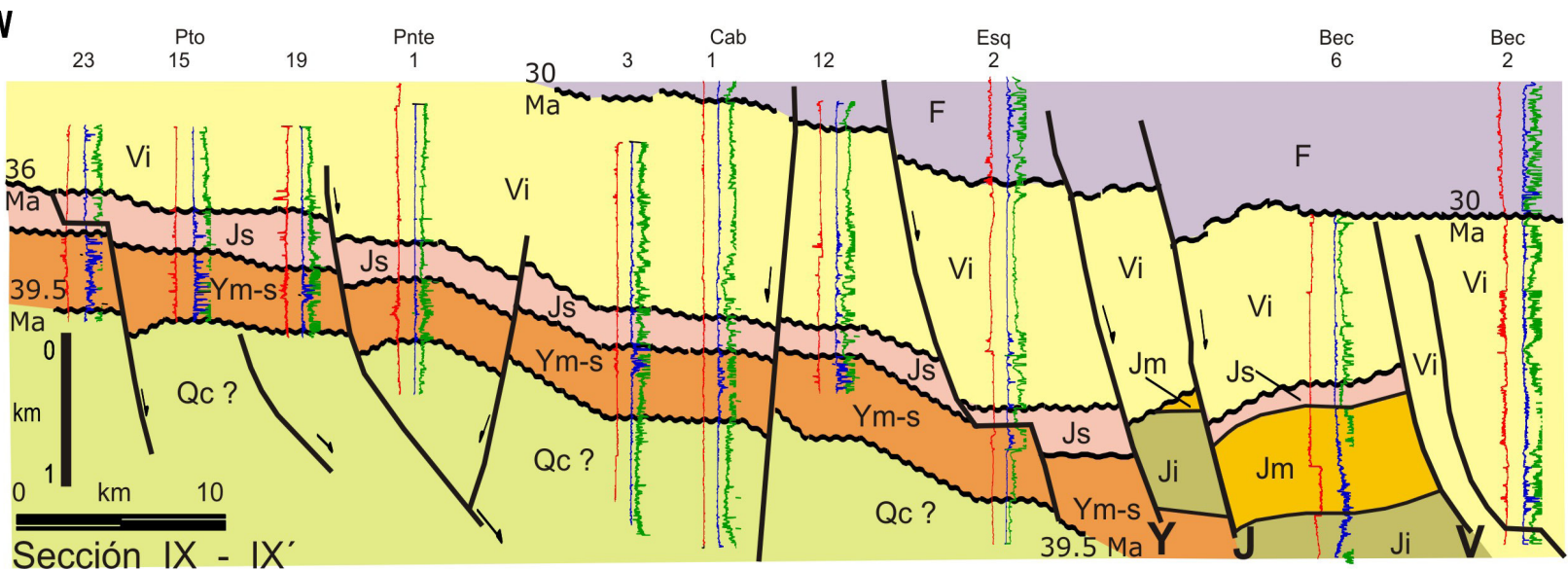

Figura 5. Referirse a la leyenda de la Figura 3.

que el límite inferior de Yegua corresponde a una litología de arenisca granocreciente, limitada en su cima por una discordancia que puede seguirse regionalmente. La base de este cuerpo arenoso inicia sobre la arcilla de la Formación Cook Mountain; el conjunto de ambas litologías corresponde a una secuencia granocreciente, progradante, de nivel alto (Highstand Systems Tract, HST), que cierra el ciclo genético, con su límite de secuencia en la parte superior, dado por una discordancia regional. Con apoyo de foraminíferos, en el presente trabajo se asume que esta discordancia puede corresponder aproximadamente a 39.5 Ma. Asimismo, se considera que este cuerpo de arenisca granocreciente es estratigráficamente equivalente a la Formación Crockett y se propone que sea denominada unidad Yegua inferior.

Con apoyo en el registro paleontológico, estudio de núcleos de barrenos, afloramientos y distribución de arenas en los bloques estructurales, se elaboró el modelo de depósito (Figura 6).

La discordancia de $39.5 \mathrm{Ma}$, hacia el oriente de las secciones, echado abajo en la cuenca, corta a capas más antiguas $\mathrm{y}$, de occidente a oriente, llega a eliminar gradualmente a sedimentos de Yegua inferior, Cook Mountain, Weches e incluso a la cima de Queen City. La discordancia 39.5 Ma es una marca regional y un límite de secuencia muy constante en toda el área (Figura 7), pero sólo en la porción central y occidente de ésta, es posible controlar su paso, ya que hacia el oriente, por las fallas de mayor crecimiento ( $\mathrm{Y}, \mathrm{J}$ y V), no puede controlarse esta discordancia a profundidad.

Yegua intermedia es un depósito de espesor delgado al poniente $(250 \mathrm{~m})$, pero se engrosa al oriente en las proximidades de la falla de expansión Q, y en la falla Y llega a alcanzar más de $700 \mathrm{~m}$ (Figura 8). Esta cuña está compuesta principalmente por material arcilloso con cuerpos de areniscas erráticas de difícil correlación distribuidas en su base como abanicos de piso y derrumbes; tiene sobrelapamiento (onlap) sobre la discordancia de 39.5 $\mathrm{Ma}$ (Figura 9). La parte media y la cima de esta sucesión de estratos de Yegua intermedia presentan numerosos canales 


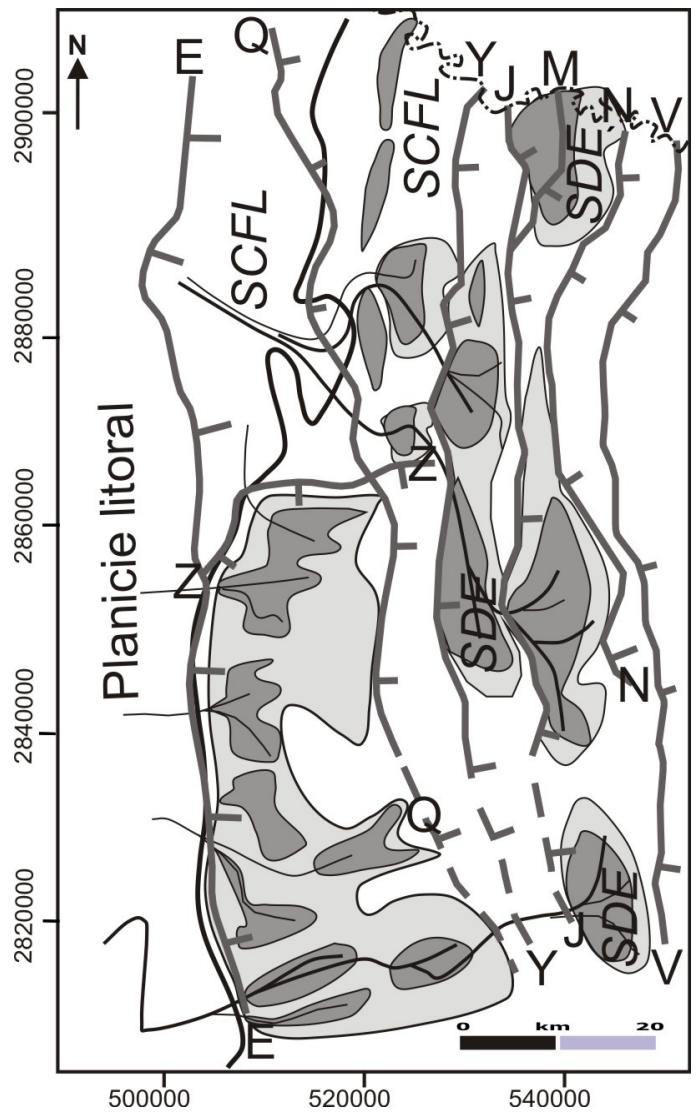

Figura 6. Modelo sedimentario y distribución de ambientes de la Formación Yegua.

o valles de incisión (Figuras 3 y 4) con varias discordancias internas observadas con sísmica, algunas de las cuales tienen continuidad por decenas de kilómetros, pero son difíciles de seguir de una sección a otra.

Sobre la litología de Yegua intermedia, en la cima de Yegua y abajo de los estratos de Jackson inferior (con control litológico y paleontológico), se presenta una sección, mayormente arenosa granocreciente, que en este trabajo se denomina como Yegua superior. El espesor de este cuerpo arenoso es de aproximadamente $250 \mathrm{~m}$.

Por la dificultad para individualizar a las unidades Yegua intermedia y superior, en este trabajo se les muestra agrupadas en las secciones regionales (Figuras 3, 4 y 5). Las unidades Yegua intermedia y superior se profundizan al oriente por efecto de fallas de crecimiento, pero hacia el sur del área de estudio su cima está erosionada profundamente y queda cubierta en discordancia por sedimentos de la Formación Jackson superior (Figuras 3, 4 y 5). En esta posición no es posible diferenciar a la Formación Yegua en unidad superior o unidad intermedia, pero se puede inferir que estos depósitos no pertenecen a Yegua inferior, ya que están descansando sobre la discordancia de $39.5 \mathrm{Ma}$, debida a la esosión de rocas de las formaciones Cook Mountain, Weches, Queen City o más antiguas (Figura 5). Hacia el norte del área, el contacto entre Yegua y Jackson inferior es

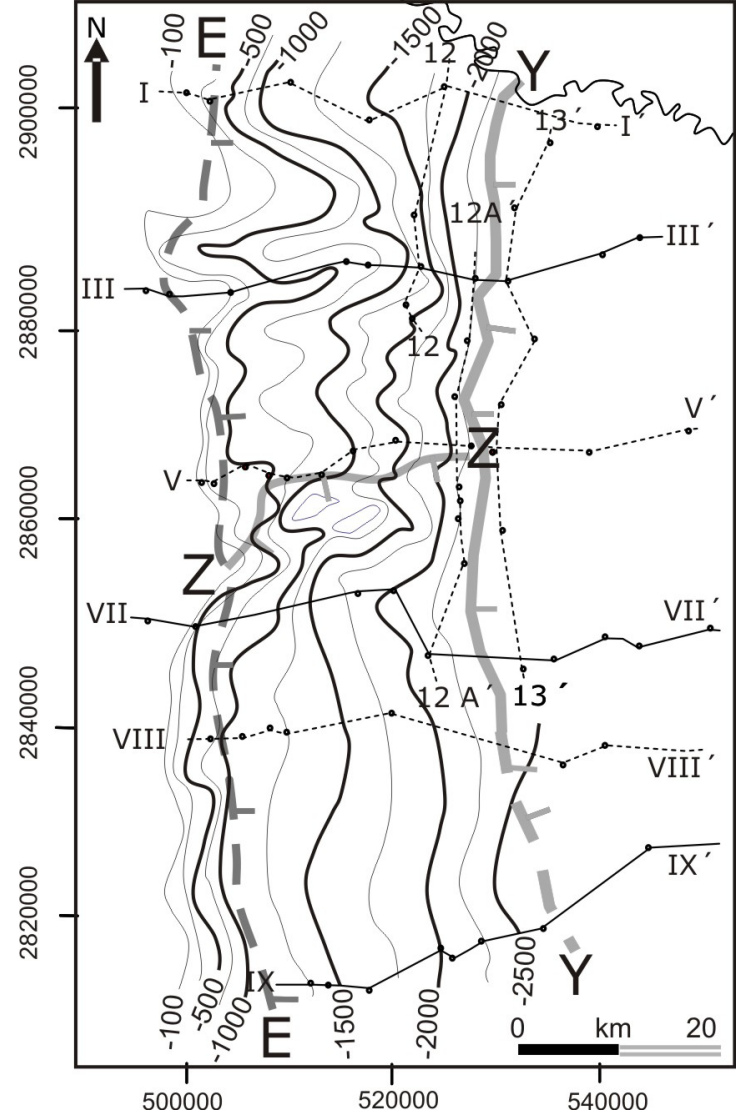

Figura 7. Mapa del contorno de la discordancia $39.5 \mathrm{Ma}$, referido en metros bajo el nivel del mar. E, Y y Z corresponden a la traza de fallas (en tono gris) como límites de la interpretación del control estructural. Los números romanos indican secciones construidas a rumbo. Los números arábigos indican secciones construidas a echado. Las secciones mostradas en este trabajo se presentan con línea continua; otras secciones (no mostradas) se indican con líneas discontinuas.

concordante, marcado por un cambio litológico y controlado por su contenido paleontológico, mientras que al sur del área, la Formación Jackson superior descansa en contacto erosivo adelgazando a Yegua intermedia.

La Formación Yegua en la parte sur del área es muy compleja. A partir de la falla regional $\mathrm{Z}$, existe un cambio sedimentológico, estratigráfico y estructural drástico, afectado por profundas erosiones, controladas posiblemente por fallas o levantamiento estructural. La correlación se dificulta aún más por la sísmica 2D con poca resolución.

\section{Análisis de secuencias}

Varios autores han analizado las secuencias depositadas en el intervalo Yegua de Texas y Luisiana (Edwards, 1990; Hull, 1995; Luneau et al., 1995; Meckel y Galloway, 1996; Ewing y Vincent, 1997a,b; Ewing, 2007). Esos autores reconocen superficies de inundación dentro de la Formación Yegua, que interpretan como marcas que pueden seguirse por decenas de kilómetros en la Planicie Costera 


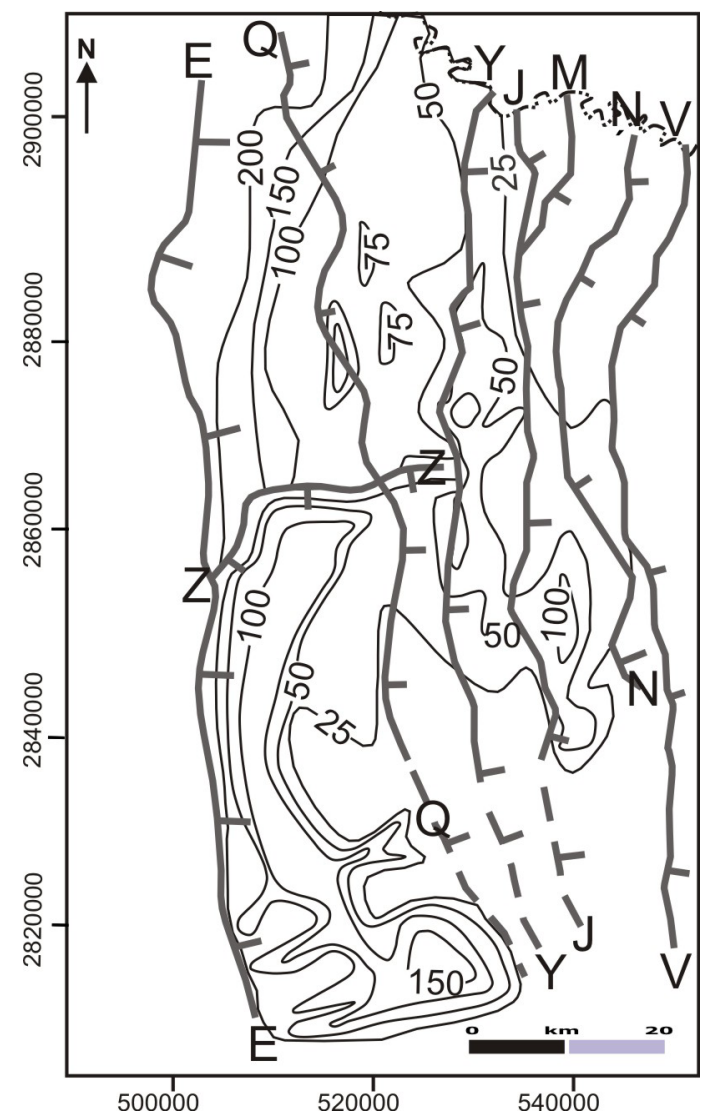

Figura 8. Distribución de espesores de arenas en Yegua inferior y Yegua intermedia. Se muestran las fallas principales (Q, E, Y, J, V, M, N, Z) que controlaron los depósitos arenosos; con línea gris cortada su traza inferida.

del Golfo de México. Edwards (1990) describió numerosos ciclos de alta frecuencia en las formaciones Yegua y Cook Mountain, y sugiere que estos ciclos pudieran corresponder a parasecuencias. Algunos ciclos los interpretó como efectos de deslizamientos controlados por gravedad (Ciclos C), y otros ciclos controlados por eustasia (Ciclos B). Edwards (1990), propone que el conjunto sedimentario tiene control tectónico (Ciclos A) y sugiere que algunas superficies discordantes dentro de la Formación Yegua corresponden a cañones, o bien fueron formadas por deslizamientos de gravedad en la plataforma continental (Kim et al., 2003). Adicionalmente, Edwards (1990) y Meckel y Galloway (1996) sugieren que la mayoría de las discordancias no son continuas y, por lo tanto, tienen limitado uso como marcas regionales, mientras que las superficies de inundación encuentran consistencia para su correlación regional en todo Texas y Luisiana, con excepción del condado de Oak, Texas (Ewing y Vincent, 1997b).

Estas correlaciones de secuencias en Texas ayudan a reconocer diferentes ambientes de sedimentación. Echado arriba de la cuenca, las facies de plataforma presentan deltas, pero las facies de frente deltaico están desplazadas echado abajo, hacia aguas profundas de la cuenca, y quedan separadas por zonas de bypass. Meckel y Galloway (1996)
Eguiluz

reconocen en Texas varias superficies de inundación, que son marcas regionales que separan ciclos de depósito de cuarto orden. Esos autores proponen que los intervalos 1 y 2 (Yegua inferior) y 4 (Yegua intermedia) constituyen secuencias en donde predomina un mayor aporte de sedimentos que el espacio de acomodo. El espacio de acomodo domina en el intervalo 6 de su correlación (cima de Yegua) y los intervalos pre-Yegua ocurrieron bajo condiciones dominadas por un bajo aporte de sedimentos y bajo nivel del mar, mientras que hay condiciones de equilibrio en los intervalos 3 y 5 , que subyacen a los intervalos 4 y 6 .

En la Cuenca de Burgos hay pocos datos bioestratigráficos con la resolución adecuada para el control de secuencias estratigráficas (Rodríguez-Lozano, 1999; Segura et al., 2006). La identificación de estas secuencias se realizó mayormente en base a la sísmica de 2 y 3 dimensiones, patrones de curvas de registros de pozos, estudio de núcleos de pozos y afloramientos. De acuerdo a estas herramientas, la unidad Yegua inferior es una sucesión arenosa granocreciente a la cima. Por su facies y correlación se interpreta de ambiente litoral que progradó al oriente y constituye una estadía de nivel alto (Highstand Systems Tract, HST). Su contacto inferior es una transición con las arcillas de Formación Cook Mountain, mientras que su contacto superior está truncado por una discordancia regional (Figuras 3, 4, 5, 7 y 9). Los datos paleontológicos indican que Yegua inferior corresponde a la base de la biozona P 14 y se correlaciona con la Formación Crockett (ahora en desuso).

La discordancia sobre Yegua inferior es un límite de secuencia (Sequence Boundary, SB) prominente, por posición estratigráfica entre las unidades Yegua inferior y Yegua intermedia, que la limitan (Figuras 2 y 9). La discordancia se correlaciona con el límite de secuencia de 39.5 Ma propuesto por Haq et al. (1988), considerado como un límite de segundo orden. Esta discordancia al occidente erosionó las cimas de la unidad Yegua inferior y la Formación Cook Mountain, al oriente erosionó unidades tan antiguas como las Formaciones Reklaw y Queen City, mientras que al sur y suroeste erosionó unidades más antiguas que las citadas.

La Formación Yegua intermedia se ubica sobre la superficie de erosión propuesta en este trabajo y arriba citada. Yegua intermedia es una sucesión de estratos que corresponden a una unidad predominantemente arcillosa, marcada en la firma geofísica de registros de pozos por una curva irregular y valores de rayos gama altos, y con gran complejidad sedimentaria por sus discordancias y deformación internas. Esta secuencia presenta areniscas de piso de cuenca, erráticos cuerpos de areniscas en canales y slumps. Se considera en su base como un depósito en etapa de nivel bajo (Lowstand Systems Tract, LST), seguido por una etapa transgresiva (Transgressive Systems Tract, TST). Su base tiene cuñas (onlap) que cubren a estratos de Yegua inferior cortados por la discordancia. Yegua intermedia puede contener secuencias de tercer orden vistas 


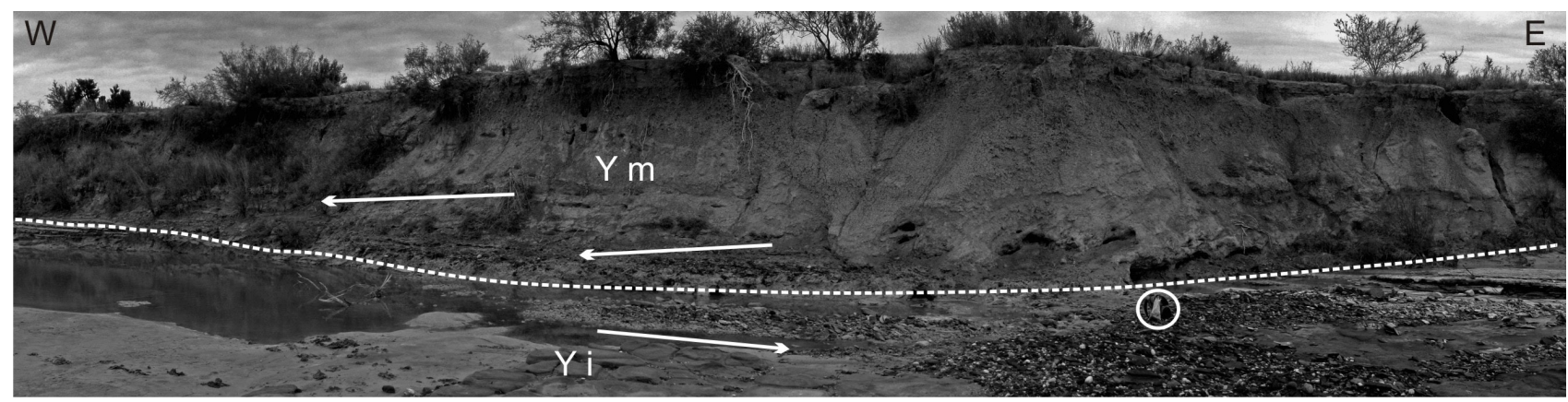

Figura 9. Discordancia entre Yegua inferior (Y i) y Yegua intermedia (Y m) en el arroyo Brasil, a $3 \mathrm{~km}$ al occidente del poblado Brasil, Nuevo León. Yegua inferior consta de capas en arenisca, de 40 a $60 \mathrm{~cm}$ de espesor, con rizaduras, estratificación festonada y estructuras de corte por canales, depositada en facies litorales, subyace truncada por una discordancia paralela (línea cortada) a Yegua intermedia, compuesta por una brecha subangulosa en su base, con estratificación cruzada y descrecencia de grano a la cima, con cuñas de sobrelapamiento (onlap) sobre la discordancia (nótese la diferencia de inclinación de estratos entre ambas unidades). Mochila como escala en la circunferencia indicada en la fotografía.

en sísmica y de cuarto orden en núcleos y afloramientos, pero en este trabajo no se alcanzó esta resolución mayor, como ha sido identificada en Texas por otros autores (Ewing y Vincent, 1997b; Edwards, 1990; Meckel y Galloway, 1996). La cima de Yegua superior no es del todo nítida, puede ser concordante o discordante según su posición con respecto a la polaridad sedimentaria de la cuenca. Cuando Yegua superior subyace a Jackson inferior generalmente se presenta granocreciente, su firma geofísica permite reconocerla y en este trabajo se denomina como Yegua superior. Esta unidad litológicamente es predominantemente arenosa, depositada en ambiente litoral, pero no siempre está bien definida, por lo que hay incertidumbre de que corresponda a un depósito de nivel alto (HST) o a un depósito transgresivo (TST), en transición hacia el miembro arcilloso de la Formación Jackson inferior, que se identifica como una zona de inundación.

Las correlaciones de Meckel y Galloway (1996) desde Texas a la Cuenca de Burgos no son del todo claras, pero en base a su trabajo se puede hacer preliminarmente el intento de considerar que Yegua inferior de este trabajo es correlacionable con los intervalos 1 y 2 de esos autores. La unidad Yegua intermedia de este escrito (un complejo de varias secuencias de frecuencia alta) pudiera ser correlativo a los intervalos de 3 a 5 y, por lo tanto, Yegua superior sería correlativa con el intervalo 6 de esos autores.

Ewing y Vincent (1997b) proveen un marco regional lógico de correlación, que indica la interrelación entre múltiples límites de secuencias y superficies de inundación para la Formación Yegua en Texas. Ese trabajo muestra un límite de secuencia ubicado alrededor de 39.5 Ma que subyace a un depósitos de cuña progradante asociado con abanicos de piso y sistemas de margen de plataforma. La zonificación de biofacies y variaciones ambientales mostradas por Ewing (2007) son similares a las que ocurren en la Cuenca de Burgos. La correlación preliminar entre las secuencias de ambas áreas sugieren (a) que el tramo entre la cima de Ceratobulimia eximia y el límite de secuencia Y-50 puede ser un depósito correlativo con los estratos de
Yegua inferior de este trabajo, (b) que sobre Yegua inferior hay un límite de secuencia regional, que en este trabajo puede situarse aproximadamente en $39.5 \mathrm{Ma}$, y (c) que los estratos situados sobre esta discordancia corresponden con las marcas entre Y-40 y Y-10 de Ewing (2007) y son correlacionables con la las unidades intermedia y superior de la Formación Yegua de este trabajo.

\section{Deformación estructural}

Hull (1995) indicó que los depósitos de Yegua en Texas tienen tasas de sedimentación promedio de $350 \mathrm{~m}(164 \mathrm{ft})$ por millón de años, los cuales son muy altos comparados con las tasas de depósitos de otras unidades del Eoceno Medio y Superior, pero comparables a la tasa de sedimentación de los depósitos del Eoceno Inferior y Paleoceno. Rosen et al. (1994) observaron también este rasgo. En la Cuenca de Burgos, los espesores de Yegua, parcialmente erosionada, son delgados echado arriba de la cuenca, mientras que echado abajo los espesores son gruesos, con tasas promedio de 280 m (919ft) por millón de años. Hull (1995) atribuye la tasa de sedimentación alta a factores climáticos. Galloway et al. (2000) proponen que el depósito de la Formación Yegua fue controlado por pulsos tectónicos del levantamiento de la Sierra Madre Oriental, apoyándose en los estudios de Yurewicz et al. (1997), que consideraron al incremento de calor del manto con actividad ígnea como evidencia. Chávez-Cabello (2005) indica que la deformación Laramide de la Cuenca de Sabinas terminó en el Eoceno y aporta datos estructurales e isotópicos $\left({ }^{40} \mathrm{Ar} /{ }^{39} \mathrm{Ar}\right.$ en hornblenda y biotita) que sugieren que la deformación ocurrió entre 44 Ma y no menos de $39.97 \pm 0.04$ Ma. Gray et al. (2001) proponen que la exhumación de la cubierta sedimentaria pudo ocurrir en el Oligoceno, edad del emplazamiento del Cinturón Magmático del Este de México, coincidente con el levantamiento del Arco del Salado (Eguiluz, 2007).

El límite de secuencia propuesto aquí, alrededor de $39.5 \mathrm{Ma}$, es una importante discordancia regional que, 
junto con la compleja sedimentación de Yegua intermedia, tentativamente pudiera ser relacionado con la edad de la deformación Laramide (Eguiluz, 2004, 2007) y el depósito de la Formación Yegua, reflejar cambios sedimentarios por tectónica, o la combinación de factores climáticos y tectónicos. Las mayores dificultades para establecer una aproximación mejor en la Cuenca de Burgos son las discrepancias que existen entre edades absolutas y edades relativas usadas para ubicar a las formaciones estratigráficas, la carencia de datos paleontológicos con mayor resolución, las definiciones litoestratigráficas inapropiadas y la escala geocronológica y su resolución según las escalas usadas por diversos autores.

\section{Conclusiones}

En este trabajo se analizó la estratigrafía de la Formación Yegua en la Cuenca de Burgos y se propone dividir a esta formación en tres unidades. Yegua inferior corresponde a una secuencia de nivel alto (HST), con su cima cortada por una discordancia regional, que corresponde a un límite de secuencia situado alrededor de $39.5 \mathrm{Ma}$.

Yegua intermedia es una unidad estratigráfica compleja, con discordancias internas, canales, deslizamientos de sedimentos y abanicos de piso marino; por posición estratigráfica está situada sobre el límite de secuencia de 39.5 Ma y forma un sistema de nivel bajo y transgresivo complejo. Se reconoce como Yegua superior a una secuencia posiblemente de nivel alto (HST) que subyace al miembro inferior de la Formación Jackson. Cuando no se reconoce esta secuencia de nivel alto, se dificulta hacer la diferenciación de las unidades Yegua intermedia y Yegua superior.

Con base en el análisis de secuencias y con apoyo de núcleos de pozos, afloramientos y espesores, se propone un modelo sedimentario para la Formación Yegua. El modelo se aproxima a definir ambientes de planicie costera, litorales, de abanico de delta y de estuario, de facies someras al occidente y profundas al oriente.

Se identificó una discordancia regional prominente como límite de secuencia de segundo orden por las características litológicas y edades de las unidades que la limitan, así como el entorno geológico regional. Se propone que el depósito de la Formación Yegua puede reflejar la etapa final de edad de deformación Laramide. Estudios adicionales de otras unidades estratigráficas en la Cuenca de Burgos son necesarios para confirmar este postulado.

\section{Agradecimientos}

Gracias al Dr. Juan Montalvo Arieta y al Dr. Gabriel Chávez Cabello por invitar al autor a participar con la publicación de este trabajo, para incrementar el conocimiento geológico del noreste de México. A Octavio Pola, Gabriel Ramos, Javier Solano, Angélica Tristán y Lawrence Meckel, que colaboraron en el estudio del los plays Yegua y Jackson. A los revisores, Margarita Livas Vera, Valente Ricoy Páramo, Alberto Segura Treviño, Andrés Boni Noguez y María Chapela, que con su conocimiento y entusiasmo orientaron al autor para mejorar el contenido de lo aquí expuesto. El autor agradece al editor Antoni Camprubi i Cano por sus acertados comentarios y esmerada revisión de este trabajo. A Petróleos Mexicanos se agradece el permiso para difundir el conocimiento geológico de nuestro país.

\section{Referencias}

Berggren, W.A., Kent, D.V., Swisher III, C.C., Aubry, M.P., 1995, A revised Cenozoic geochronology and chronostratigraphy, en Berggren, W.A., Kent, D.V., Aubry, M.P., Hardenbol, J. (eds.), Geochronology, time scales and global stratigraphic correlation: Tulsa, Oklahoma, SEPM (Society for Sedimentary Geology), 129-212.

Chávez-Cabello, G., 2005, Deformación y magmatismo cenozoico en el sur de la Cuenca de Sabinas, Coahuila, México: Juriquilla, Querétaro, Centro de Geociencias, Universidad Nacional Autónoma de México, tesis doctoral, $266 \mathrm{p}$.

Deussen, A., 1914, Geology and underground waters of the southeastern part of the Texas Coastal Plain: Washington, D.C., Government Printing Office, $365 \mathrm{p}$.

Dumble, E.T., 1892, Report of the Brown Coal and Lignite of Texas. Character, Formation, Occurrence, and Fuel Uses: Austin, Texas, Geological Survey of Texas, 243 p.

Dumble, E.T., 1894, The Cenozoic deposits of Texas: Journal of Geology, 2, 549-567.

Eargle, D.H., 1968, Nomenclature of formations of Claiborne Group, middle Eocene, coastal plain of Texas: U. S. Geological Survey Bulletin, 1251-D, 1-25.

Edwards, M.B., 1990, Stratigraphic analysis and reservoir prediction in the Eocene Yegua and Cook Mountain formations of Texas and Louisiana, Gulf Coast, en GCSSEPM Foundation 11th Annual Research Conference Program and Abstracts: Houston, Texas, Gulf Coast Section SEPM (Society for Sedimentary Geology), 1-14.

Eguiluz, S., 2004, Tectónica Laramide en la Cuenca de Burgos, México: Revista GEOS, 24, 307-308.

Eguiluz, S., 2007, Laramide deformation in the Burgos Basin, Northeastern Mexico, en 27th Annual GCSSEPM Foundation Bob F. Perkins Research Conference: Houston, Texas, Gulf Coast Section SEPM (Society for Sedimentary Geology), 688-702.

Ewing, T.E., 2007, Fairways in the downdip and middip Yegua trend; a review of 25 years of exploration: Transactions - Gulf Coast Association of Geological Societies, 57, 227-248.

Ewing, T.E., Vincent, F.S., 1997a, Foundered shelf edges - Examples from the Yegua and Frio, Texas and Louisiana: Transactions - Gulf Coast Association of Geological Societies, 47, 149-157.

Ewing, T.E., Vincent, F.S., 1997b, Correlation of Yegua/Cockfield genetic cycles, Texas and Louisiana: Transactions - Gulf Coast Association of Geological Societies, 47, 631-633.

Galloway, W.E., Ganey-Curry, P.E., Li, X., Buffler, R.T., 2000, Cenozoic depositional history of the Gulf of Mexico Basin: AAPG Bulletin, 84, 1743-1774.

Gray, G.G., Pottorf, R.J., Yurewicz, D.A., Mahon, K.I., Pevear, D.R., Chuchla, R.J., 2001, Thermal and chronological record of syn- to post-Laramide burial and exhumation, Sierra Madre Oriental, Mexico, en Bartolini, C., Buffler R.T., Cantú-Chapa, A. (eds.), The western Gulf of Mexico Basin: Tectonics, Sedimentary Basins, and Petroleum Systems: Tulsa, Oklahoma, American Association of Petroleum Geologists, 159-181. 
Haq, B.U., Hardenbol, J., Vail, P.R., 1988, Mesozoic and Cenozoic chronostratigraphy and eustatic cycles, en Wilgus, C.K., Hastings, B.S., Posamentier, H., van Wagoner, J., Ross, C.A., Kendall, C.G.S.C. (eds.), Sea-level changes: an integrated approach: Tulsa, Oklahoma, Society of Economic Paleontologists and Mineralogists, 71-108.

Holroyd, P.A., 2002, New record of anthracotheriidae (Artiodactyla: Mammalia) from the middle Eocene Yegua Formation (Claiborne Group), Houston County, Texas: The Texas Journal of Science, 54, 301-308.

Hull, R.A., 1995, Shelf-slope break sedimentation and erosion during deposition of the Claiborne Group in Colorado and Wharton counties, Texas: Transactions - Gulf Coast Association of Geological Societies, 45, 267-274.

Kennedy, W., 1892, A section from Terrell, Kaufman County, to Sabine Pass on the Gulf of Mexico, en Dumble, E.T. (ed.), Third Annual Report of the Geological Survey of Texas, 1891: Austin, Texas, Geological Survey of Texas, 41-125.

Kennedy, W., 1895, The Eocene Tertiary of Texas east of the Brazos River: Proceedings of the Academy of Natural Sciences of Philadelphia, 47, 86-160.

Kim, J.W., Berg, R.R., Watkins, J.S., Tieh, T.T., 2003, Trapping capacity of faults in the Eocene Yegua Formation, East Sour Lake field, southeast Texas: AAPG Bulletin, 87, 415-425.

Lagoe, M.B., Layman, T.B., 1994, Comprehensive, quantitative micropaleontological analysis as a tool for paleoenvironmental interpretation and sequence stratigraphy, with an example from the Yegua Formation, southeast Texas: AAPG Bulletin, 78, 1464.

Luneau, B.A., Eschner, T.G., Gomez, Scott, A.J., 1995, Sequencestratigraphic framework of the Yegua Formation in the Houston Salt Basin (resumen), en AAPG Annual Convention: Houston, Texas, American Association of Petroleum Geologists, 58.

Luterbacher, H.P., Ali, J.R., Brinkhuis, H., Gradstein, F.M., Hooker, J.J., Monechi, S., Ogg, J.G., Powell, J., Rühl, U., Sanfilippo, A., Schmitz, B., 2004, The Paleogene Period, en Gradstein, F.M., Ogg, J.G., Smith, A.G. (eds.), A Geologic Time Scale 2004: Cambridge, Reino Unido, Cambridge University Press, 384-408.

Meckel, L.D., Galloway, W.E., 1996, Formation of high-frequency sequences and their bounding surfaces: Case study of the Eocene Yegua Formation, Texas, Gulf Coast, USA: Sedimentary Geology, 102, 155-186.
Penrose, R.A.F., 1890, A preliminary report on the geology of the Gulf Tertiary of Texas from Red River to the Rio Grande, en Dumble, E.T. (ed.), First Annual Report of the Geological Survey of Texas: Austin, Texas, Geological Survey of Texas, 3-101.

Plummer, F.B., 1932, Cenozoic Systems in Texas, en Sellards, E.H. Adkins, W.S., Plummer, F.B. (eds.), The geology of Texas, Volume I, Stratigraphy: Austin, Texas, The University of Texas Bulletin, 519-996.

Rodríguez-Lozano, D.E., 1999, Bioestratigrafía de la franja PaleocenoEoceno de la Cuenca de Burgos, en Meneses Rocha, J., Velazco Vázquez, G., Rosales Domínguez, M.C. (eds.), La Cuenca de Burgos: Villahermosa, Tabasco, Asociación Mexicana de Geólogos Petroleros, 227-239.

Rosen, R.N., Bowen, B.E., Thies, K.J., 1994, Subsurface planktonic zonation of the Paleogene of Texas and Louisiana Gulf Coast and its relationship to relative changes of coastal onlap, en Transactions of the Gulf Coast Association of Geological Societies 44th Annual Convention: Austin, Texas, 631-639.

Segura Treviño, A., Fuentes, J.N., Ramírez Villaseñor, G., González García, A.V., Guerra Pérez, S., Quintanilla Pérez, M., 2006, Carta Bioestratigráfica de la Cuenca de Burgos (cartel), en Simposio de Paleontología: Reynosa, Tamaulipas, Asociación Mexicana de Geólogos Petroleros.

Swenson, R.D., 1997, Basal Yegua shelf margin failures along the Texas Gulf Coast: Transactions - Gulf Coast Association of Geological Societies, 47, 571-577.

Vaughan, T.W., 1896, The stratigraphy of northwestern Louisiana: The American Geologist, 15, 205-229.

Yurewicz, D. A., Chuchla, R.J., Richardson, M., Pottorf, R.J., Gray, G., Kozar, M.G., Fitchen, W.M., 1997, Hydrocarbon generation and migration in the Tampico- Misantla Basin and Sierra Madre Oriental, east central Mexico: Evidence from an exhumed oil in the Sierra de El Abra, en Sedimentation and diagenesis of middle Cretaceous platform margins, east central Mexico: AAPG Annual Meeting Field Trip Guidebook, Dallas Geological Society and SEPM, 1-24 p.

Manuscrito recibido: Abril 16, 2009.

Manuscrito corregido recibido: Julio 24, 2010.

Manuscrito aceptado: Octubre 19, 2010. 